\title{
Loss of $13 q$ is associated with genes involved in cell cycle and proliferation in dedifferentiated hepatocellular carcinoma
}

\author{
Britta Skawran ${ }^{1}$, Doris Steinemann ${ }^{1}$, Thomas Becker ${ }^{2}$, Reena Buurman ${ }^{1}$, Jakobus Flik ${ }^{3}$, \\ Birgitt Wiese $^{4}$, Peer Flemming ${ }^{5}$, Hans Kreipe ${ }^{5}$, Brigitte Schlegelberger ${ }^{1}$ and \\ Ludwig Wilkens ${ }^{1,5}$
}

\begin{abstract}
${ }^{1}$ Institute of Cell and Molecular Pathology, Hannover Medical School, Hannover, Germany; ${ }^{2}$ Department of Visceral and Transplantation Surgery, Hannover Medical School, Hannover, Germany; ${ }^{3}$ Institute of Virology, Hannover Medical School, Hannover, Germany; ${ }^{4}$ Institute of Biometry, Hannover Medical School, Hannover, Germany and ${ }^{5}$ Institute of Pathology, Hannover Medical School, Hannover, Germany
\end{abstract}

\begin{abstract}
Dedifferentiation of hepatocellular carcinoma implies aggressive clinical behavior and is associated with an increasing number of genomic alterations, eg deletion of $13 q$. Genes directly or indirectly deregulated due to these genomic alterations are mainly unknown. Therefore this study compares array comparative genomic hybridization and whole genome gene expression data of 23 well, moderately, or poorly dedifferentiated hepatocellular carcinoma, using unsupervised hierarchical clustering. Dedifferentiated carcinoma clearly branched off from well and moderately differentiated carcinoma $\left(P<0.001 \chi^{2}\right.$-test). Within the dedifferentiated group, 827 genes were upregulated and 33 genes were downregulated. Significance analysis of microarrays for hepatocellular carcinoma with and without deletion of $13 q$ did not display deregulation of any gene located in the deleted region. However, 531 significantly upregulated genes were identified in these cases. A total of 6 genes (BIC, CPNE1, RBPMS, RFC4, RPSA, TOP2A) were among the 20 most significantly upregulated genes both in dedifferentiated carcinoma and in carcinoma with loss of 13q. These genes are involved in cell-cycle control and proliferation. Of 33 downregulated genes in the dedifferentiated subgroup, 4 metallothioneins had the lowest fold change, most probably mediated through inactivation of C/EBP $\alpha$ by the PI3K/AKT cascade. In conclusion dedifferentiation of hepatocellular carcinoma is associated with upregulation of genes involved in cell-cycle control and proliferation. Notably, a significant portion of these genes is also upregulated in carcinoma with deletion of 13q. As no downregulated genes were identified and microRNAs (mir-621, mir16-1, mir-15a) are located within the deleted region of $13 q$ and may be lost, we speculate that these miRNAs may induce the upregulation of critical cell-cycle control genes.
\end{abstract}

Modern Pathology (2008) 21, 1479-1489; doi:10.1038/modpathol.2008.147; published online 26 September 2008

Keywords: hepatocellular carcinoma; gene expression; chromosomal instability; microRNA; cell cycle; proliferation

Sustained basic, clinical, and epidemiological studies have previously been performed to address the major problems caused by the fourth most malignant tumor in the world: hepatocellular carcinoma $^{1}$ (http://www-dep.iarc.fr/). In many previous studies, this has led to the detection of various mutations of genes (eg p53, IGF2R, p16, PTEN) typically associated with the development of hepatocellular carcinoma. ${ }^{2}$ Besides these specific mutations, the

Correspondence: Professor L Wilkens, MD, Institute of Pathology, University of Bern, Murtenstrasse 31, Postfach 62, Bern CH 3010, Switzerland.

E-mail: ludwig.wilkens@pathology.unibe.ch

Received 13 June 2008; revised 16 July 2008; accepted 19 July 2008; published online 26 September 2008 carcinogenesis of hepatocellular carcinoma is known to be correlated with telomere shortening ${ }^{3}$ and chromosomal instability. ${ }^{4}$

Chromosomal instability is defined by microscopically detectable partial or complete gain or loss of chromosomes typically occurring in hepatocellular carcinoma and also in many other solid tumors. ${ }^{5,6}$ The typical set of chromosomal imbalances found in comparative genomic hybridization studies includes gains of 1q, 6p, 8q, 17q, and 20q, and losses of 1p, 4q, $6 q, 8 p, 13 q, 16 q$, and $17 p$ detected in $>90 \%$ of 780 cases analyzed up to now (see references 7-10 for review: $\left.{ }^{11}\right)$. These aberrations have been correlated with etiological factors such as viral (hepatitis B, hepatitis C) infections. ${ }^{12-14}$ Clinical impact has been reported for losses of $8 p$ and gain of $20 q$ with respect 
Table 1 Clinical, morphological, and serological data of the 23 patients analyzed

\begin{tabular}{|c|c|c|c|c|c|c|c|}
\hline No. & Age & Gender & $\begin{array}{l}\text { pTNM } \\
\text { classification }\end{array}$ & Diameter & $\begin{array}{l}\text { Morphological } \\
\text { grading }\end{array}$ & $\begin{array}{l}\text { Hepatitis } \\
\text { serology }^{\mathrm{a}}\end{array}$ & ISHAK-score \\
\hline $\mathrm{N} 20$ & 68 & $\mathrm{~F}$ & $\mathrm{pT} 2, \mathrm{NX}, \mathrm{MX}$ & 5 & $\mathrm{~W}$ & Neg & NA \\
\hline N25 & 54 & $\mathrm{M}$ & pT2, N0, MX & 1.5 & W & B & A1B0C1D2F6 \\
\hline N77 & 53 & $\mathrm{~F}$ & pT3, NX, MX & 18 & W & Neg & No cirrhosis \\
\hline N81 & 43 & $\mathrm{M}$ & pT2, NX, MX & 7 & W & Neg & No cirrhosis \\
\hline N1 & 10 & M & pT2, No, MX & 2.5 & M & Neg & A1B0C0D1F6 \\
\hline N8 & 63 & M & $\mathrm{pT} 2, \mathrm{NX}, \mathrm{MX}$ & 9 & $\mathrm{M}$ & $\mathrm{C}$ & A4B0C2D4F2 \\
\hline N9 & 81 & $\mathrm{M}$ & $\mathrm{pT} 2, \mathrm{NX}, \mathrm{MX}$ & 9 & $\mathrm{M}$ & B & No cirrhosis \\
\hline N13 & 65 & $\mathrm{~F}$ & pT4, NX, MX & 14 & $\mathrm{M}$ & Neg & No cirrhosis \\
\hline N15 & 68 & M & pT3, NX, M1 & 6.5 & $\mathrm{M}$ & Neg & A2B0C1D2F6 \\
\hline N37 & 71 & $\mathrm{M}$ & $\mathrm{pT} 2, \mathrm{NX}, \mathrm{MX}$ & 6 & $\mathrm{M}$ & Neg & No cirrhosis \\
\hline N62 & 75 & M & pT3, N0, MX & 2.5 & $\mathrm{M}$ & Neg & A2B0C1D1F \\
\hline N69 & 73 & $\mathrm{M}$ & pT2, NX, MX & 2.5 & $\mathrm{M}$ & Neg & A2B0C1D2F4 \\
\hline N74 & 35 & $\mathrm{M}$ & pT2, NX, MX & 6 & $\mathrm{M}$ & Neg & No cirrhosis \\
\hline N80 & 65 & $\mathrm{M}$ & pT3, NX, MX & 9 & $\mathrm{M}$ & B & No cirrhosis \\
\hline N87 & 47 & $\mathrm{M}$ & pT2, NX, MX & 3.3 & $\mathrm{M}$ & $\mathrm{C}$ & A3B0C1D3F6 \\
\hline N45 & 73 & $\mathrm{~F}$ & pT2, NX, MX & 10 & $\mathrm{~L}$ & $\mathrm{C}$ & A2B0C1F2 \\
\hline N47 & 65 & $\mathrm{M}$ & pT3, NX, MX & 8 & $\mathrm{~L}$ & B & A1B0C1D1F2 \\
\hline N71 & 59 & M & pT3, NX, MX & 17 & $\mathrm{~L}$ & B & No cirrhosis \\
\hline N86 & 57 & $\mathrm{M}$ & pT3, NX, MX & 8 & $\mathrm{~L}$ & Neg & No cirrhosis \\
\hline N90 & 9 & $\mathrm{M}$ & pT3, NX, M1 & 3 & $\mathrm{~L}$ & B & No cirrhosis \\
\hline N91 & 37 & M & pT2, NX, MX & 15 & $\mathrm{~L}$ & B & A1B0C1D2F0 \\
\hline N40 & 50 & $\mathrm{M}$ & pT3, N0, MX & 11 & $\mathrm{~L}$ & B & No cirrhosis \\
\hline N82 & 19 & M & pT3, No, MX & 4 & $\mathrm{~L}$ & B & A1B0C1D1F6 \\
\hline
\end{tabular}

B/C: serological evidence of hepatitis B/C; NA: not available.

${ }^{\mathrm{a}} \mathrm{Neg}$ : no serological evidence of hepatitis B and/or C.

to stage and prognosis. ${ }^{15}$ On the basis of genomic alterations detected by aCGH, hepatocellular carcinoma and its benign counterpart, hepatocellular adenoma, could be differentiated. ${ }^{16}$ It was also noted that dedifferentiation of hepatocellular carcinoma was closely correlated to losses of $4 \mathrm{q}$ and $13 \mathrm{q} \cdot{ }^{11}$ By fluorescence in situ hybridization, a stepwise increase in genomic instability, as well as a highly significant correlation of histological grading and grade of polyploidization, was seen. ${ }^{17}$ This is of clinical interest because morphological grading has been shown to correlate with survival. ${ }^{18,19}$ Elucidation of the processes causing dedifferentiation of hepatocellular carcinoma is required because hepatocellular carcinoma dedifferentiated in more than half of patients within 7-34 months..$^{20,21}$

Investigations into the genomic and mRNA expression level have been attempted to provide insight into these processes, but a combined analysis has not yet been carried out. Using newly developed software tools for the analysis of genomic array data, we therefore aimed to identify genes deregulated during dedifferentiation of hepatocellular carcinoma, particularly those located in chromosomal regions known to be altered in poorly differentiated hepatocellular carcinoma.

\section{Materials and methods}

Clinical, serological, and pathomorphological data of 23 patients with hepatocellular carcinoma treated at Hannover Medical School between 1998 and 2002 are given in Table 1 . Tumor specimens from these patients were analyzed with regard to mRNA expression in hepatocellular adenoma in an earlier study. ${ }^{22}$ Tumor material was obtained as surgical specimens taken for diagnostic purposes as permitted by the Ethics Committee of Hannover Medical School. Unfixed tissue samples of $0.5 \mathrm{~cm}$ in diameter were snap-frozen within $30 \mathrm{~min}$ after resection and stored at $-80^{\circ} \mathrm{C}$. A part of the tissue was cut out for RNA extraction; another piece was fixed in formalin and embedded in paraffin for histological examination. This ensured the closest proximity and homogeneity of the tissues used for different experiments.

Histological classification according to WHO criteria, ${ }^{23}$ was carried out after $\mathrm{H} \& \mathrm{E}$ and reticulin staining by two experienced pathologists (L.W., P.F.). A total of 4 well, 11 moderately, and 8 poorly differentiated hepatocellular carcinoma were detected with histological signs of partial or complete dedifferentiation (Figure 1).

For 22/23 cases of hepatocellular carcinoma, cytogenetic characterization was already performed using aCGH; detailed information is given in an earlier study. ${ }^{16}$ The findings described according to the international system of cytogenetic nomenclature ISCN ${ }^{24}$ are given in Supplementary Table 1. In brief, the most commonly gained region was 1q22.1-23.1 in 20 cases (91\%). Gains of $8 q$ were observed in 14 cases (64\%), losses of $8 \mathrm{p}$ in 11 cases $(50 \%)$, gains of $20 \mathrm{q}$ in 9 cases 

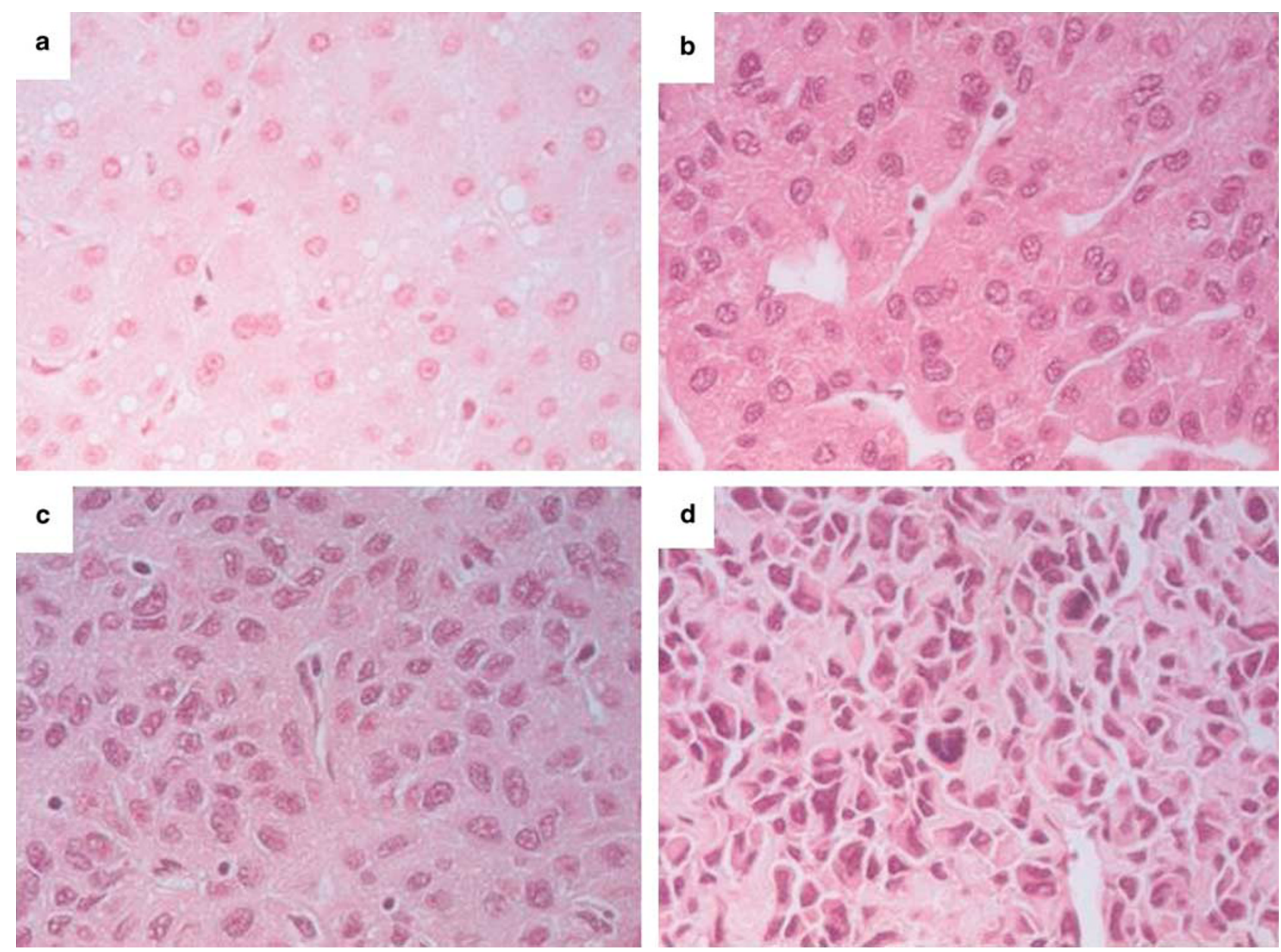

Figure 1 Histological differentiation of hepatocellular carcinoma showing cytological and architectural features. (a) Well-differentiated hepatocellular carcinoma is defined by nuclei shape, which is very similar to normal hepatocytes. Additional staining (eg silver staining) may be required to ensure correct diagnosis. (b) Moderately differentiated carcinoma reveals a heterogeneous pattern with respect to cytology of nuclei and architectural pattern. (c) In moderate-to-poorly differentiated hepatocellular carcinoma, prominent nucleoli are seen in the nuclei; the shape of the nuclei and membrane is irregular. The trabecular pattern is also disturbed in a prominent way, making recognition of trabecular structures difficult. (d) In dedifferentiated hepatocellular carcinoma, nuclei have a bizarre and highly irregular shape, and lose their architectural pattern, making it difficult to separate these carcinomas from dedifferentiated carcinomas of other histogenetic origin.

(41\%), losses of $13 q$ in 9 cases (41\%), losses of $4 q$ in 8 cases $(36 \%)$, losses of $6 q$ in 8 cases (36\%), gains of $6 p$ in 7 cases $(32 \%)$, and losses of $17 p$ in 7 cases (32\%).

\section{Methods}

cDNA microarray analysis

cDNA microarray experiments were carried out as previously described. ${ }^{22}$ In brief, total RNA was isolated using Trizol (Invitrogen, Karlsruhe, Germany) followed by RNeasy Mini Kit (Qiagen, Hilden, Germany) and linearly amplified (MessageAmp aRNA Kit; Ambion, Huntingdon, UK). Aliquots of $1.5 \mu \mathrm{g}$ of amplified RNA from the tumors and the human reference were reverse transcriptase labeled with Cy3 (reference) and Cy5 (tumor) (Amersham Biosciences, Little Chalfont, UK). Labeled RNA of tumors and references were com- paratively hybridized overnight onto a cDNA chip containing the 'Resgen clone set' with $>43000$ spots covering the entire human genome (Stanford Functional Genomics Facility, Stanford, CA, USA) according to the posted protocol (http://brownlab. Stanford.edu/protocols.html). Arrays were imaged using a GenePix 4000B scanner and dependent software (GenePix Pro 4.1; Axon Instruments). Data were exported to the Stanford Microarray Database (http://smd.stanford.edu). Fluorescence ratios were normalized for each array. Data were excluded if the regression correlation was $<0.6$, or the mean spot intensity/median background intensity was $<1.5$. The measured genes were subsequently 'meancentered' (ie reported for each gene relative to the mean ratio across all samples, rather than relative to the human reference).

The hierarchical clustering algorithm based on Pearson correlation (centered) coefficients grouped 

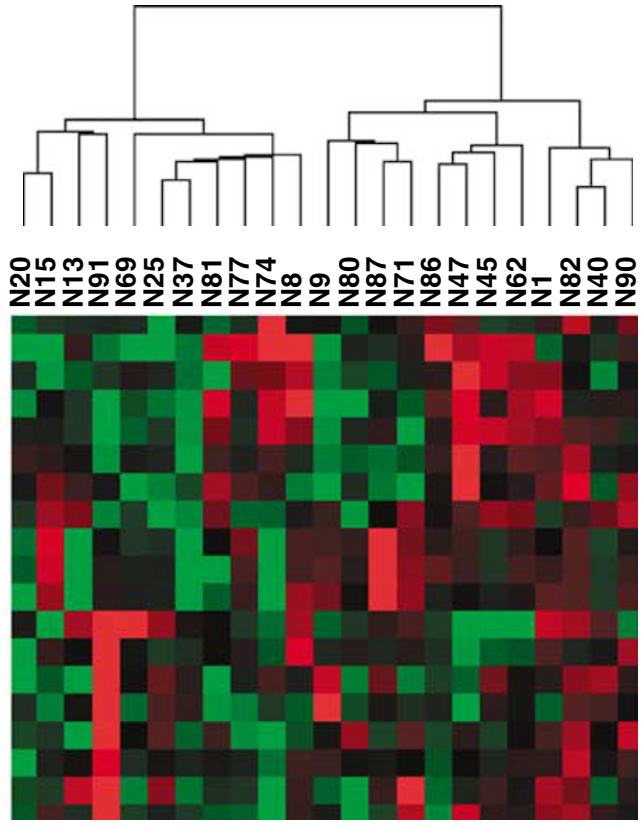

wmml mw mwwmmmml I I I mm l I I

Grade of differentiation

w: well differentiated carcinoma

$\mathrm{m}$ : moderately differentiated carcinoma

I: low differentiated carcinoma

Figure 2 Unsupervised hierarchical clustering of global gene expression analysis. Upregulated genes are marked in red, downregulated genes in green. Two groups of hepatocellular carcinoma were obtained using this approach, with all welldifferentiated carcinoma clustering in group A and all lowdifferentiated carcinoma clustering in group B with only one exception (N91). Moderately differentiated carcinomas were found in both clusters. Carcinomas of these two groups were analyzed in regard to histological grading and $13 q-$.

the genes (as well as the samples) on the basis of similarity in their expression pattern. Normalized and log-transformed expression data served as input for significance analysis of microarrays ${ }^{25}$ with standard parameters. False discovery rates varied between 0.0 and 0.54 .

$R T-P C R$

Quantitative RT-PCR was performed on the selected genes MT1F, MT1G, and TOP2A, to confirm the array results.

In a total volume of $120 \mu \mathrm{l}, 1 \mu \mathrm{g}$ of total RNA was reverse transcribed by $\mathrm{M}-\mathrm{MuLV}$ reverse transcriptase using random hexamer primers (RevertAid First Strand cDNA Synthesis Kit; Fermentas, St-Leon-Rot, Germany). TOP2A cDNA samples $(2 \mu \mathrm{l})$ were amplified on an iCycler iQ real-time detection system (Bio-Rad Laboratories, München, Germany) using a QuantiTect SYBR Green PCR kit (Qiagen, Hilden, Germany). TBP was used as the internal control 


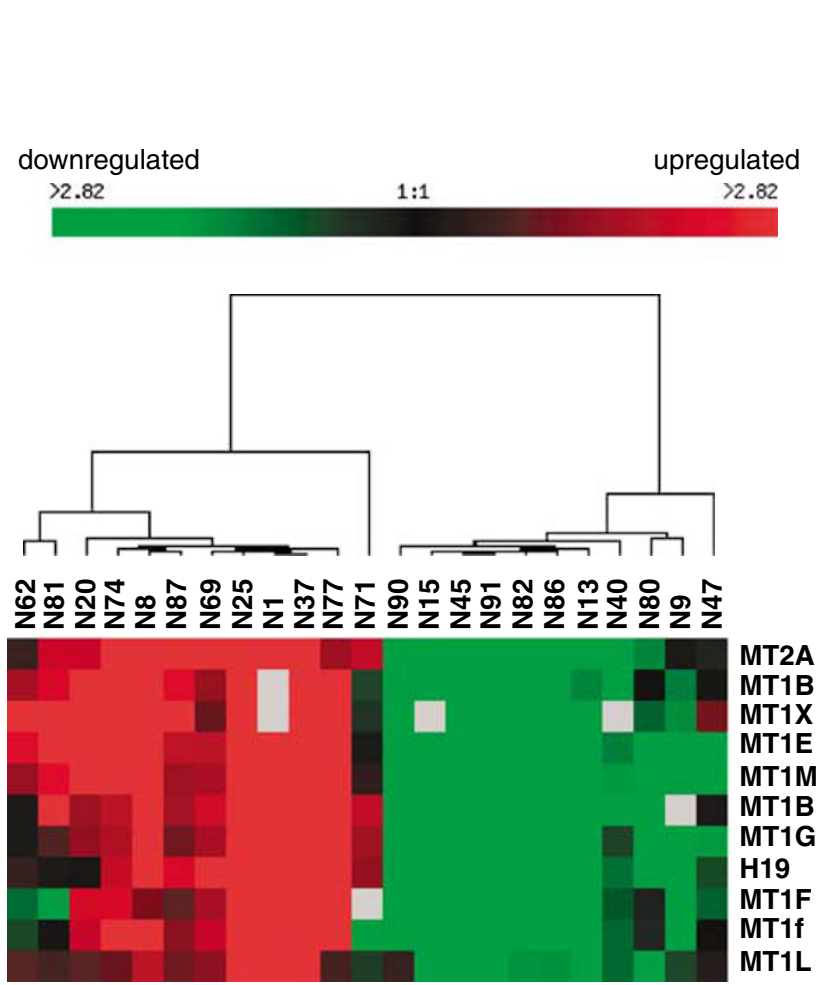

mww mmmmwmm w | | m I | | | | m I m m ।

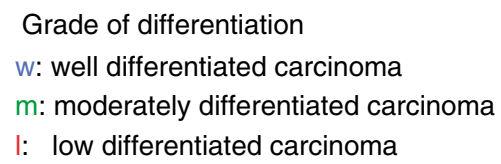

Figure 3 Supervised cluster analyses for members of the metallothionein family branched off all dedifferentiated carcinoma from well and moderately differentiated tumors. Welldifferentiated carcinomas are grouped together in one group, whereas low-differentiated carcinoma are in the other group. Upregulated genes are marked in red, downregulated genes in green.

gene. Aliquots of $5 \mu \mathrm{l}$ of each cDNA sample were used for the analysis of $M T 1 F$ and $M T 1 G$ expression. This expression was performed using an iCycler iQ real-time detection system (Bio-Rad Laboratories). Primers and probes for $M T 1 F$ and $M T 1 G$ were synthesized by TibMolBiol (Berlin, Germany). Detailed PCR conditions and primer sequences for all genes used are shown in Table 2. $\beta$-Actin was used as the internal control gene. For calibration, the human reference (Stratagene, Amsterdam, Netherlands) was used. Relative quantification of gene expression was determined according to the $\Delta \Delta$-CT method using an iCycler iQ real-time detection system software version 3.1 (Bio-Rad Laboratories). All samples were analyzed in triplicate.

\section{Results}

\section{cDNA Microarray Analysis}

Gene expression analysis led to successful evaluation of all 23 cases; 7977 cDNA clones with the most varying expression among the tumor and reference samples were selected for further bioinformatical analysis.
$13 q$ - in hepatocellular carcinoma

B Skawran et al

\section{Hierarchical Clustering}

Unsupervised hierarchical clustering separated hepatocellular carcinoma into two groups of 11 and 12 cases. All well-differentiated carcinoma were seen in the first group. In the second group, all lowdifferentiated carcinoma occurred, with the exception of case N91. In the former group, six moderately differentiated carcinoma were seen, whereas five moderately differentiated carcinoma occurred in the latter (Figure 2).

Statistical analysis was further performed by correlation with histological grading. For this parameter, well and moderately differentiated carcinoma were taken together (15 cases) and compared to lowdifferentiated carcinoma (8 cases). A highly significant correlation $\left(P<0.001, \chi^{2}\right.$-test $)$ was detectable in regard to the histological grading. For example, four metallothioneins (MT1E, MT1F, MT1M, MT2A) had the lowest fold change $(0.09-0.18)$ in poorly differentiated hepatocellular carcinoma. Supervised cluster analyses for these genes branched off all dedifferentiated tumors from well and moderately differentiated samples (Figure 3).

\section{Analysis of Gene Expression by Statistical Analysis of Microarrays}

Using this approach for 'histological grading', the number of significantly dysregulated genes with a false discovery rate of $<5 \%$ was 860 . A total of 827 genes were upregulated in the group of dedifferentiated carcinoma; 33 genes were downregulated (Supplementary Tables 2 and 3). For loss of 13q, the number of dysregulated genes was 531 .

For further analysis of these findings, we focused on 20 genes seen with the highest or lowest fold changes (Table 3). For histological grading, the fold change of the most upregulated 20 genes was 2.587.47. For the 20 downregulated genes, this change was $0.09-0.47$. For the parameter loss of 13q, the top 20 upregulated genes had fold changes of 2.62-4.00. Only two genes (MDFIC and GALE) were downregulated, with fold changes of 0.38 and 0.40 , respectively.

Six genes were found in both sets of upregulated genes, one from dedifferentiated carcinoma and one from carcinoma with 13q- (BIC, CPNE1, RBPMS, RFC4, RPSA, TOP2A). All of these genes mentioned are involved in cell-cycle control and proliferation. Supervised cluster analyses for these genes, branched off all dedifferentiated carcinoma from well and the moderately differentiated carcinoma. Furthermore, these analyses branch off the most carcinoma with $13 q$ - from carcinoma without $13 q-$ (Figure 4 ).

In the sets of genes downregulated in poorly differentiated carcinoma, four metallothioneins (MT1E, MT1F, MT1M, MT2A) had the lowest fold change (0.09-0.18). All metallothioneins are allocated to chromosome band 16q13. A loss of 16q13 may have caused the downregulation in three 
Table 3 SAM in regard to histological grading and loss of 13q

\begin{tabular}{|c|c|c|c|}
\hline Parameter: & Histological grading & & \\
\hline Symbol & $\begin{array}{c}\text { Fold } \\
\text { change }\end{array}$ & Cytoband & Name/function \\
\hline
\end{tabular}

\begin{tabular}{|c|c|c|c|c|}
\hline \multicolumn{5}{|c|}{ Upregulated genes } \\
\hline CDK7 & 7.47 & 1.05 & $5 q 12.1$ & $\begin{array}{l}\text { Cyclin-dependent kinase } 7 \text { (MO15 homolog, Xenopus laevis, } \\
\text { cdk-activating kinase) }\end{array}$ \\
\hline STAM2 & 3.80 & 2.58 & $2 q 23.3$ & Signal transducing adapter molecule (SH3 domain and ITAM motif) 2 \\
\hline TOP2A & 3.58 & 0.00 & $17 q 21-q 22$ & Topoisomerase (DNA) II alpha $170 \mathrm{kDa}$ \\
\hline SPINT2 & 3.47 & 3.64 & $19 \mathrm{q} 13.1$ & Serine peptidase inhibitor, Kunitz type, 2 \\
\hline CDCA7 & 3.40 & 1.29 & 2q31 & Cell division cycle associated 7 \\
\hline$B I C$ & 3.21 & 1.29 & $21 q 21.3$ & B-cell receptor inducible gene \\
\hline OSTM1 & 3.03 & 4.34 & $6 q 21$ & Osteopetrosis associated transmembrane protein 1 \\
\hline RFC4 & 3.01 & 0.00 & $3 q 27$ & Replication factor C (activator 1) 4, $37 \mathrm{kDa}$ \\
\hline$R B P M S$ & 2.96 & 1.05 & $8 p 12-p 11$ & RNA binding protein with multiple splicing \\
\hline KPNA2 & 2.91 & 0.00 & $17 q 24.2$ & Karyopherin- $\alpha 2$ (RAG cohort 1 , importin alpha 1 ) \\
\hline ATAD2 & 2.87 & 0.00 & $8 q 24.13$ & ATPase family, AAA domain containing 2 \\
\hline RPSA & 2.75 & 0.66 & 3p22.2 & Ribosomal protein SA \\
\hline DDR1 & 2.72 & 4.34 & $6 \mathrm{p} 21.3$ & Discoidin domain receptor family, member 1 \\
\hline CKS2 & 2.72 & 0.00 & $9 q 22$ & CDC28 protein kinase regulatory subunit 2 \\
\hline MCM6 & 2.70 & 0.00 & $2 q 21$ & Minichromosome maintenance complex component 6 \\
\hline MCM3 & 2.70 & 0.00 & $6 p 12$ & Minichromosome maintenance complex component 3 \\
\hline NAP1L1 & 2.69 & 0.66 & $12 \mathrm{q} 21.2$ & Nucleosome assembly protein 1 -like 1 \\
\hline CPNE1 & 2.61 & 1.29 & $20 \mathrm{q} 11.22$ & Copine I \\
\hline ARL4A & 2.60 & 1.05 & 7p21-p15.3 & ADP-ribosylation factor-like $4 \mathrm{~A}$ \\
\hline NANS & 2.58 & 0.00 & $9 \mathrm{p} 24.1-\mathrm{p} 23$ & $N$-acetylneuraminic acid synthase (sialic acid synthase) \\
\hline
\end{tabular}

Symbol $\begin{gathered}\text { Fold } \\ \text { change }\end{gathered}$ FDR Cytoband Name

\begin{tabular}{lllll}
\hline \multicolumn{2}{l}{$\begin{array}{l}\text { Downregulated genes } \\
\text { MT1E }\end{array}$} & & & \\
MT1F & 0.09 & 2.58 & $16 \mathrm{q} 13$ & Metallothionein 1E \\
MT1M & 0.10 & 3.64 & $16 \mathrm{q} 13$ & Metallothionein 1F (pseudogene) \\
MT2A & 0.18 & 3.64 & $16 \mathrm{q} 13$ & Metallothionein 1M \\
GHR & 0.19 & 0.00 & $5 \mathrm{p} 13-\mathrm{p} 12$ & Metallothionein 2A \\
SORL1 & 0.27 & 5.01 & $11 \mathrm{q} 23.2-\mathrm{q} 24.2$ & Growth hormone receptor \\
AGXT2L1 & 0.28 & 0.69 & $4 \mathrm{q} 25$ & Sortilin-related receptor, L (DLR class) A repeats-containing \\
SLC27A2 & 0.31 & 3.64 & $15 \mathrm{q} 21.2$ & Alanine-glyoxylate aminotransferase 2-like 1 \\
SORBS2 & 0.36 & 0.00 & $4 \mathrm{q} 35.1$ & Solute carrier family 27 (fatty acid transporter), member 2 \\
CYP3A5 & 0.37 & 2.58 & $7 \mathrm{q} 21.1$ & Sorbin and SH3 domain containing 2 \\
SLC23A2 & 0.38 & 5.01 & $20 \mathrm{p} 13$ & Cytochrome P450, family 3, subfamily A, polypeptide 5 \\
HRSP12 & 0.40 & 3.64 & $8 \mathrm{q} 22$ & Solute carrier family 23 (nucleobase transporters), member 2 \\
MGC119295 & 0.44 & 0.00 & $7 \mathrm{q} 22.1$ & Heat-responsive protein 12 \\
FNBP1 & 0.44 & 3.64 & $9 \mathrm{q} 34$ & Similar to Williams-Beuren syndrome critical region protein 19 \\
CHD9 & 0.45 & 4.34 & $16 \mathrm{q} 12.2$ & Formin binding protein 1 \\
RCL1 & 0.46 & 4.34 & $9 \mathrm{p} 24.1-\mathrm{p} 23$ & Chromodomain helicase DNA-binding protein 9 \\
GSTK1 & 0.46 & 5.01 & 7 & RNA terminal phosphate cyclase-like 1 \\
BDH2 & 0.47 & 3.64 & $4 \mathrm{q} 24$ & Glutathione $S$-transferase-k1 \\
PON1 & 0.47 & 5.01 & $7 \mathrm{q} 21.3$ & 3-Hydroxybutyrate dehydrogenase, type 2 \\
HIPK2 & 0.47 & 3.64 & $7 \mathrm{q} 32-\mathrm{q} 34$ & Paraoxonase 1 \\
& & & & Homeodomain-interacting protein kinase 2
\end{tabular}

\begin{tabular}{|c|c|c|c|c|}
\hline \multirow{2}{*}{$\begin{array}{l}\text { Parameter: } \\
\text { Symbol }\end{array}$} & \multicolumn{2}{|c|}{ Loss of $13 q$} & \multirow[b]{2}{*}{ Cytoband } & \multirow[b]{2}{*}{ Name } \\
\hline & $\begin{array}{l}\text { Fold } \\
\text { change }\end{array}$ & $F D R$ & & \\
\hline \multicolumn{5}{|c|}{ Upregulated genes } \\
\hline IL1RN & 4.00 & 1.58 & $2 q 14.2$ & Interleukin 1 receptor antagonist \\
\hline RPL29 & 3.38 & 1.58 & 3p21.3-p21.2 & Ribosomal protein L29 \\
\hline ARMCX3 & 3.32 & 1.45 & $\mathrm{Xq} 21.33-\mathrm{q} 22.2$ & Armadillo repeat containing, X-linked 3 \\
\hline RPSA & 3.18 & 0.00 & $3 \mathrm{p} 22.2$ & Ribosomal protein SA \\
\hline EIF4A1 & 3.02 & 1.45 & $17 \mathrm{p} 13$ & Eukaryotic translation initiation factor 4A, isoform 1 \\
\hline SMARCA4 & 2.95 & 1.45 & $19 \mathrm{p} 13.2$ & $\begin{array}{l}\text { SWI/SNF-related, matrix-associated, actin-dependent regulator of } \\
\text { chromatin, subfamily A }\end{array}$ \\
\hline RBPMS & 2.94 & 3.16 & 8p12-p11 & RNA-binding protein with multiple splicing \\
\hline$B I C$ & 2.94 & 3.16 & $21 q 21.3$ & Neuroblastoma cDNA, clone: Nbla00208, full insert sequence \\
\hline CPNE1 & 2.82 & 1.58 & $20 q 11.22$ & Copine I \\
\hline C1orf151 & 2.82 & 0.00 & $1 \mathrm{p} 36.13$ & Chromosome 1 open reading frame 151 \\
\hline
\end{tabular}


Table 3 Continued

\begin{tabular}{|c|c|c|c|c|}
\hline \multirow{2}{*}{$\begin{array}{l}\text { Parameter: } \\
\text { Symbol }\end{array}$} & \multicolumn{2}{|c|}{ Loss of $13 q$} & \multirow[b]{2}{*}{ Cytoband } & \multirow[b]{2}{*}{ Name } \\
\hline & $\begin{array}{l}\text { Fold } \\
\text { change }\end{array}$ & $F D R$ & & \\
\hline AHCYL1 & 2.76 & 0.00 & $1 \mathrm{p} 13.2$ & $\begin{array}{l}\text { Similar to adenosylhomocysteinase ( } S \text {-adenosyl-L-homocysteine } \\
\text { hydrolase) (AdoHcyase) }\end{array}$ \\
\hline$A R L 4 D$ & 2.75 & 0.00 & $17 q 12-q 21$ & ADP-ribosylation factor-like 4D \\
\hline RPS5 & 2.75 & 1.45 & $19 q 13.4$ & Ribosomal protein S5 \\
\hline TOP2A & 2.69 & 2.53 & $17 q 21-q 22$ & Topoisomerase (DNA) II alpha $170 \mathrm{kDa}$ \\
\hline$C P E$ & 2.68 & 3.16 & $4 \mathrm{q} 32.3$ & Carboxypeptidase E \\
\hline RPS7 & 2.68 & 0.00 & $2 \mathrm{p} 25$ & Ribosomal protein S7 \\
\hline WDR91 & 2.68 & 1.45 & $7 q 33$ & WD repeat domain 91 \\
\hline VDAC2 & 2.68 & 0.00 & $10 q 22$ & Voltage-dependent anion channel 2 \\
\hline RFC4 & 2.66 & 0.00 & $3 \mathrm{q} 27$ & Replication factor C (activator 1) 4, $37 \mathrm{kDa}$ \\
\hline RPS14 & 2.62 & 2.09 & $5 q 31-q 33$ & Ribosomal protein S14 \\
\hline Symbol & $\begin{array}{l}\text { Fold } \\
\text { change }\end{array}$ & $F D R$ & Cytoband & Name \\
\hline \multicolumn{5}{|c|}{ Downregulated genes } \\
\hline MDFIC & 0.38 & 2.09 & $7 q 31.1-q 31.2$ & MyoD family inhibitor domain containing \\
\hline$G A L E$ & 0.40 & 2.09 & $1 \mathrm{p} 36-\mathrm{p} 35$ & UDP-galactose-4-epimerase \\
\hline
\end{tabular}

Upregulated genes are mainly involved in cell-cycle control and proliferation, in particular six genes (marked in gray) found upregulated for both parameters.

samples (N1, N37, N83) according to the results of aCGH.

\section{Real-Time RT-PCR for Relative Quantification of TOP2A, MT1F, and MT1G}

Results obtained for mRNA expression analysis were proven exemplarily for genes TOP2A, MT1G, and MT1F. TOP2A was found upregulated in both sets, $M T 1 G$ and $M T 1 F$ were downregulated in the group of dedifferentiated hepatocellular carcinoma. RT-PCR confirmed the results of array analysis revealing a systematic increase of mRNA expression of TOP2A and a decrease for MT1G and MT1F as also found by statistical analysis of microarrays, summarized graphically in Figure 5.

\section{Comparison of Cytogenetic Data with mRNA Expression}

Because no up- or downregulated gene was located on 13q, and looking for additional factors that could be influenced by deletion of 13q, an Internet-based search using the Sanger Data Bank (www.microrna. sanger.ac.uk) was performed and identified miRNAs mir-621, mir-16-1, and mir-15a within the deleted region of 13q. As reported earlier, miRNAs can alter gene expression in a direct or nondirect way (for review see reference 25), and thus are possible candidates modulating gene expression.

\section{Discussion}

Array-based gene expression analyses on hepatocellular carcinoma have been performed to identify genes in regard to grading, staging, and etiological factors associated with clinical outcome. ${ }^{26-31}$

As $50-75 \%$ of patients develop dedifferentiation within $7-34$ months,$^{20,21}$ this has a major clinical impact due to the extremely aggressive clinical behavior of dedifferentiated hepatocellular carcinoma. ${ }^{18,19}$ We aimed to identify genes involved in the dedifferentiation of hepatocellular carcinoma. We previously showed that chromosomal instability increases parallel to dedifferentiation of hepatocellular carcinoma. ${ }^{17}$ Moreover, the number of copy number alterations increases parallel to dedifferentiation of hepatocellular carcinoma. Hepatocellular adenoma have a significantly lower number of copy number alterations than carcinoma. Hepatocellular adenoma can also be distinguished from carinoma based on global gene expression profiling. ${ }^{22} \mathrm{Com}-$ paring aCGH and gene expression data, it was possible to define a set of seven upregulated genes localized on chromosome band 1q22. As histological dedifferentiation is accompanied by loss of $13 \mathrm{q},{ }^{11,16}$ we focused on gene expression in different grades of hepatocellular carcinoma bearing $13 \mathrm{q}$ or not. We therefore aimed in this study to analyze whether well and poorly differentiated carcinoma can be distinguished based on gene expression profiling, in order to identify genes involved in the dedifferentiation of hepatocellular carcinoma.

Overall statistical analysis of microarrays analysis revealed 860 genes significantly dysregulated in dedifferentiated hepatocellular carcinoma and 533 genes related to loss of 13q. When comparing 20 genes most upregulated both during dedifferentiation and due to loss of 13q, 6 genes (BIC, CPNE1, RBPMS, RFC4, RPSA, TOP2A) were found. B-cell 
$\begin{array}{lc}\underset{72.82}{\text { downregulated }} & \text { unregulated } \\ 2.82\end{array}$
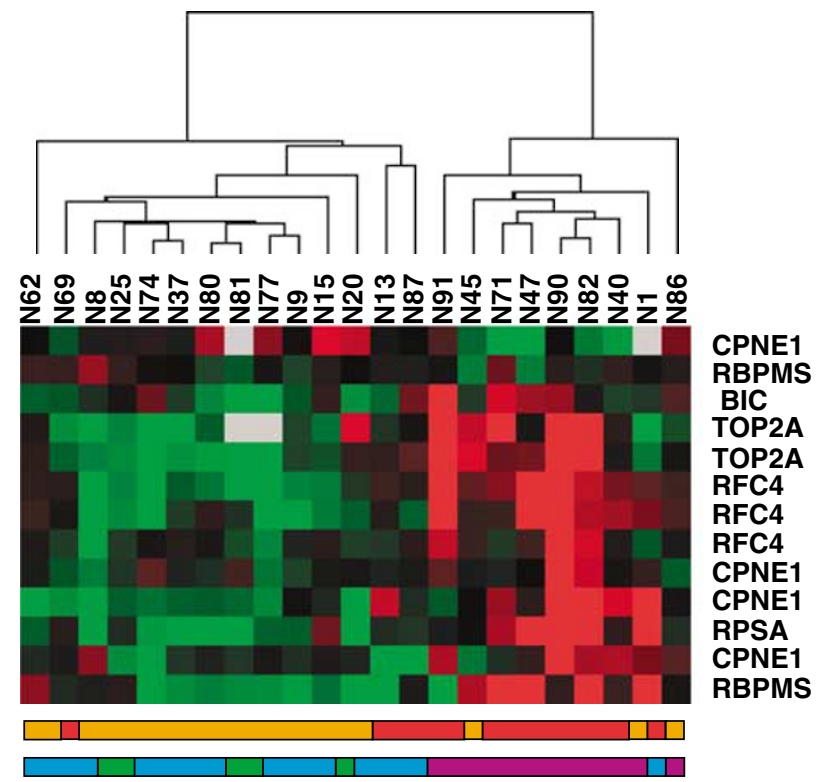

Grade of differentiation and $13 q-/ 13 q$

$\square$ carcinoma without loss of $13 q$

$\square$ carcinoma with loss of $13 q$

well differentiated carcinoma

moderately differentiated carcinoma

low differentiated carcinoma

Figure 4 Supervised clustering including six genes (BIC, CPNE1, $R B P M S, R F C 4, R P S A$, TOP $2 A$ ) branched off all dedifferentiated hepatocellular carcinoma from well and moderately differentiated carcinoma, with only one moderately differentiated hepatocellular carcinoma sample (N1) occurring in the cluster of dedifferentiated carcinoma. Vice versa, no dedifferentiated carcinoma was seen in the groups of well or moderately differentiated carcinoma. Furthermore, these analyses branched off most of carcinoma with loss of $13 q$ without $13 q-$. Upregulated genes are marked in red, downregulated genes in green.

receptor-inducible gene $(B I C)$ was originally identified as a retroviral integration site in avian leukosis virus ${ }^{32}$ and its product, miRNA 155 , accumulates in various B-cell neoplasias. ${ }^{33}$ Upregulation of $B I C$ is important in lymphomagenesis due to interaction with $C-M Y C,{ }^{34}$ which is a gene that also plays a fundamental role in hepatocarcinogenesis. CPNE1 encodes a calcium-dependent membrane-binding protein. It has a broad tissue distribution, and is thought to be involved in membrane trafficking. $R B P M S$ is a member of the RRM family of RNAbinding proteins. The protein encoded by this gene has a single, putative RRM domain, and its function is probably binding to RNA.

RPSA belongs to a family of extracellular matrix glycoproteins called laminins. These proteins have been implicated in cell adhesion, differentiation, migration, signaling, and metastasis, processes fre- quently altered in cancer cells, determining their invasive and metastatic potential. RFC4, together with PCNA, is necessary for elongation of primed DNA templates by DNA polymerase- $\delta$ and DNA

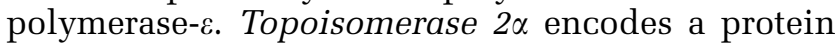
that controls and alters the topologic states of DNA during transcription. It is found in the nucleus and involved in chromosome condensation, chromatid separation, and the relief of torsional stress during the transcription and replication of DNA. It catalyzes the transient breaking and rejoining of two strands of duplex DNA, which allows the strands to pass through one another, thus altering the topology of DNA. Moreover, high Topoisomerase $2 \alpha$ expression appears to be linked with cellular dedifferentiation and potentially aggressive tumor phenotype in invasive breast cancer. ${ }^{35}$

For the genes mentioned above, more detailed information can be obtained by accessing the URLs in Supplementary Table 2.

Significantly downregulated genes were seen to be associated with dedifferentiation. Four metallothionein genes had the lowest fold change. Metallothioneins are a group of cysteine-rich intracellular proteins with high affinity for metal ions. Their enzymatic activity is not known. Metallothionein genes are located in chromosome band 16q13. In hepatocellular carcinoma, a decrease in the intensity of immunohistochemical staining has been reported for metallothioneins. ${ }^{36,37}$ Metallothioneins were also found to be downregulated in hepatocellular carcinoma in a global mRNA expression analysis by Okabe et $a l,{ }^{30}$ concordant with our results. In patients suffering from hepatocellular carcinoma, metallothioneins were measured in serum, and the concentration correlated inversely with the grade of differentiation of hepatocellular carcinoma. $^{38,39}$ In oxidative stress, eg due to infection with hepatitis-B virus or hepatitis-C virus leading to chronic inflammation, metallothioneins are reported to be cell-protective in mice. ${ }^{40}$ Conversely, MT-1 and MT-2 knockout mice are markedly more sensitive to chemically induced carcinogenesis. $^{41,42}$ Very recently, it has been shown that expression of metallothioneins is suppressed in hepatocellular carcinoma mediated through inactivation of $\mathrm{C} / \mathrm{EBP} \alpha$ by the PI3K/AKT cascade. ${ }^{43}$ Elements of this pathway (eg ErbB2, mTOR) can be modulated by various drugs ${ }^{44}$ that are probably candidates for targeted treatment of hepatocellular carcinoma.

Interestingly, none of the downregulated genes were identified within the deleted region of 13q. However, three known miRNAs (mir-621, mir-16-1, mir-15a) are located in the deleted chromosomal region of $13 q$ and therefore may be lost. This could lead to a less effective inhibition of targets of these miRNA and to an increase of gene expression of putative oncogenes, particularly on the protein level. It may also be speculated that partial loss of miRNA positively upregulates critical cell-cycle control genes such as those listed above. 

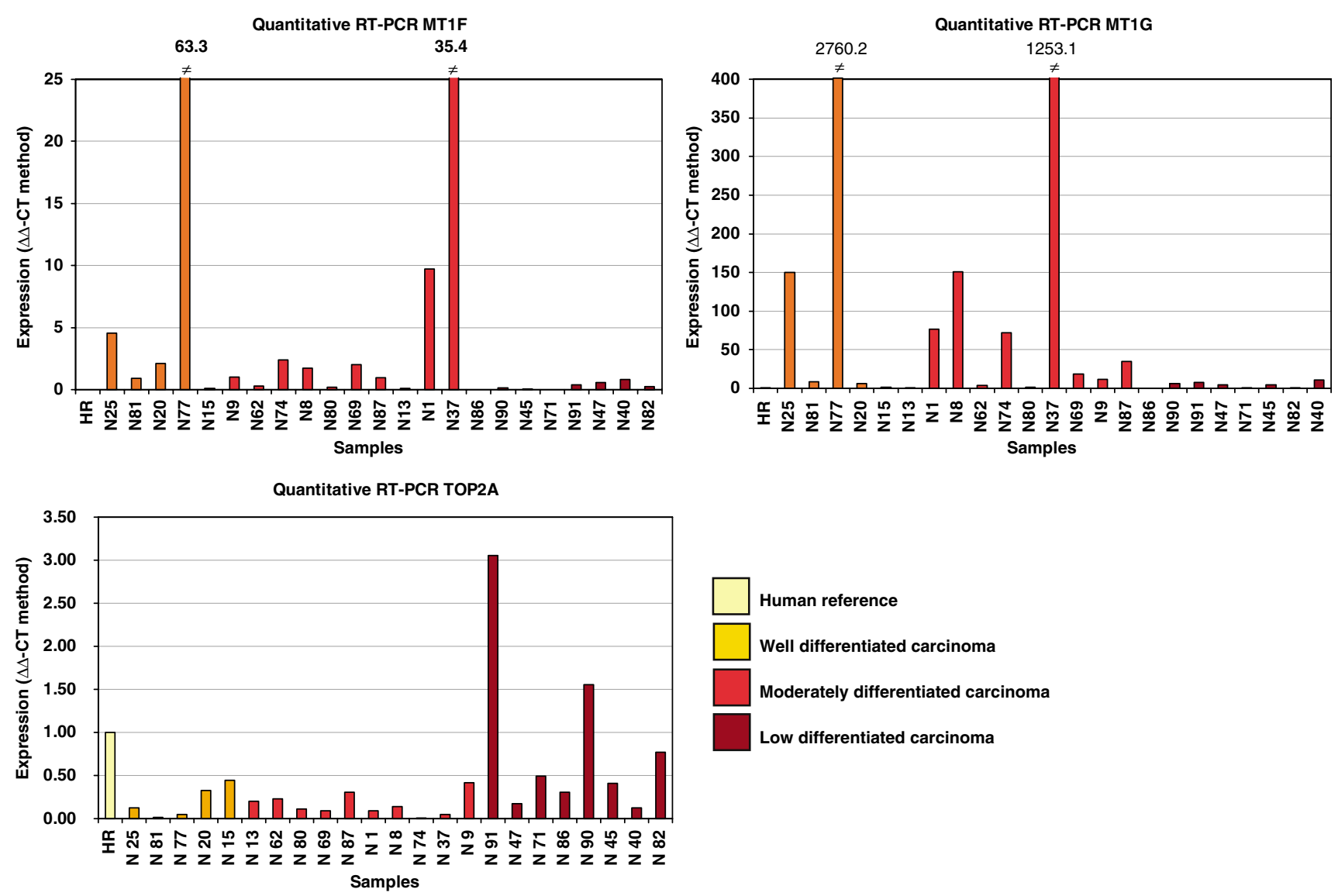

Figure 5 Graphical illustration of RT-PCR. Array-based mRNA expression analysis was confirmed by these experiments. The decreased gene expression on the mRNA level for $M T 1 F$ and $M T 1 G$ was also detectable by quantitative RT-PCR as demonstrated by significantly lower $\Delta \Delta$-CT values in low-differentiated carcinoma compared to well and moderately differentiated carcinoma. Vice versa, TOP2A was seen to be upregulated in low-differentiated carcinoma, as expected after array-based expression analysis.

In conclusion, dedifferentiation of hepatocellular carcinoma is associated with upregulation of genes involved in cell-cycle control and proliferation. Notably, a significant portion of these genes is also upregulated in hepatocellular carcinoma with deletion of 13q. It is unclear how genes located in chromosome regions other than 13q are upregulated because downregulated genes were not identified within the deleted region of 13q. As three known miRNAs (mir-621, mir-16-1, mir-15a) are located in the deleted chromosomal region of 13q, we speculate that these miRNAs are involved in dedifferentiation and in the induction of chromosomal instability in hepatocellular carcinoma and should be investigated in further studies.

\section{Acknowledgements}

This work was supported by grants from the Deutsche Forschungsgemeinschaft (KFO 119) and the Deutsche Krebshilfe (106200).

\section{Disclosure/conflict of interest}

The authors have no conflict of interest to disclose.

\section{References}

1 El-Serag HB. Hepatocellular carcinoma: an epidemiologic view. J Clin Gastroenterol 2002;35(5 Suppl 2): 72-78.

2 Thorgeirsson SS, Grisham JW. Molecular pathogenesis of human hepatocellular carcinoma. Nat Genet 2002;31:339-346.

3 Wiemann SU, Satyanarayana A, Buer J, et al. Contrasting effects of telomere shortening on organ homeostasis, tumor suppression, and survival during chronic liver damage. Oncogene 2005;24:1501-1509.

4 Plentz RR, Caselitz M, Bleck JS, et al. Hepatocellular telomere shortening correlates with chromosomal instability and the development of human hepatoma. Hepatology 2004;40:80-86.

5 Mitelman F. Catalog of Chromosome Aberrations in Cancer. 5th edn. Wiley-Liss: New York, 1994.

6 Rajagopalan H, Lengauer C. Aneuploidy and cancer. Nature 2004;432:338-341. 
7 Marchio A, Meddeb M, Pineau P, et al. Recurrent chromosomal abnormalities in hepatocellular carcinoma detected by comparative genomic hybridization. Genes Chromosomes Cancer 1997;18:59-65.

8 Wong N, Lai P, Lee SW, et al. Assessment of genetic changes in hepatocellular carcinoma by comparative genomic hybridization analysis: relationship to disease stage, tumor size, and cirrhosis. Am J Pathol 1999;154:37-43.

9 Kusano N, Shiraishi K, Kubo K, et al. Genetic aberrations detected by comparative genomic hybridization in hepatocellular carcinomas: their relationship to clinicopathological features. Hepatology 1999;29:1858-1862.

10 Wilkens L, Bredt M, Flemming P, et al. Diagnostic impact of fluorescence in situ hybridization in the differentiation of hepatocellular adenoma and welldifferentiated hepatocellular carcinoma. J Mol Diagn 2001;3:68-73.

11 Moinzadeh P, Breuhahn K, Stutzer H, et al. Chromosome alterations in human hepatocellular carcinomas correlate with aetiology and histological grade-results of an explorative CGH meta-analysis. $\mathrm{Br} \mathrm{J}$ Cancer 2005;92:935-941.

12 Laurent-Puig P, Legoix P, Bluteau O, et al. Genetic alterations associated with hepatocellular carcinomas define distinct pathways of hepatocarcinogenesis. Gastroenterology 2001;120:1763-1773.

13 Hashimoto K, Mori N, Tamesa T, et al. Analysis of DNA copy number aberrations in hepatitis $\mathrm{C}$ virusassociated hepatocellular carcinomas by conventional CGH and array CGH. Mod Pathol 2004;17: 617-622.

14 Sy SM, Wong N, Lai PB, et al. Regional overrepresentations on chromosomes $1 q, 3 q$ and $7 q$ in the progression of hepatitis B virus-related hepatocellular carcinoma. Mod Pathol 2005;6:86-92.

15 Guan XY, Fang Y, Sham JS, et al. Recurrent chromosome alterations in hepatocellular carcinoma detected by comparative genomic hybridization. Genes Chromosomes Cancer 2000;29:110-116.

16 Steinemann D, Skawran B, Becker T, et al. Assessment of differentiation and progression of hepatic tumors using array- based comparative genomic hybridization. Clin Gastroenterol Hepatol 2006; 4:1283-1291.

17 Wilkens L, Flemming P, Gebel M, et al. Induction of aneuploidy by increasing chromosomal instability during dedifferentiation of hepatocellular carcinoma. Proc Natl Acad Sci USA 2004;101:1309-1314.

18 Nzeako UC, Goodman ZD, Ishak KG. Comparison of tumor pathology with duration of survival of North American patients with hepatocellular carcinoma. Cancer 1995;76:579-588.

19 Lauwers GY, Terris B, Balis UJ, et al. Prognostic histologic indicators of curatively resected hepatocellular carcinomas: a multi-institutional analysis of 425 patients with definition of a histologic prognostic index. Am J Surg Pathol 2002;26: 25-34.

20 Kenmochi K, Sugihara S, Kojiro M. Relationship of histologic grade of hepatocellular carcinoma (HCC) to tumor size, and demonstration of tumor cells of multiple different grades in single small HCC. Liver 1987;7:18-26.

21 Sugihara S, Nakashima O, Kojiro $\mathrm{M}$, et al. The morphologic transition in hepatocellular carcinoma.
A comparison of the individual histologic features disclosed by ultrasound-guided fine-needle biopsy with those of autopsy. Cancer 1992;70:1488-1492.

22 Skawran B, Steinemann D, Weigmann A, et al. Gene expression profiling in hepatocelluar carcinoma: coordinated up-regulation of genes localised in amplified chromosome regions. Mod Pathol 2008;21: 505-516.

23 Ishak KG, Anthony PP, Sobin LH. Histological typing of tumours of the liver. World Health Organization International Histological Classification of Tumours. 2nd edn. Springer-Verlag: Berlin, 1994.

24 Shaffer LG, Tommerup N, eds. ISCN 2005: An International System for Human Cytogenetic Nomenclature. S. Karger: Basel, 2005.

25 Tusher VG, Tibshirani R, Chu G. Significance analysis of microarrays applied to the ionizing radiation response. Proc Natl Acad Sci USA 2001;98: 5116-5121.

26 Neo SY, Leow CK, Vega VB, et al. Identification of discriminators of hepatoma by gene expression profiling using a minimal dataset approach. Hepatology 2004;39:944-953.

27 Wu CG, Salvay DM, Forgues M, et al. Distinctive gene expression profiles associated with Hepatitis B virus $\mathrm{x}$ protein. Oncogene 2001;20:3674-3682.

28 Smith MW, Yue ZN, Geiss GK, et al. Identification of novel tumor markers in hepatitis $C$ virus-associated hepatocellular carcinoma. Cancer Res 2003;63:859-864.

29 Shirota Y, Kaneko S, Honda M, et al. Identification of differentially expressed genes in hepatocellular carcinoma with cDNA microarrays. Hepatology 2001;33: 832-840.

30 Okabe H, Satoh S, Kato T, et al. Genome-wide analysis of gene expression in human hepatocellular carcinomas using cDNA microarray: identification of genes involved in viral carcinogenesis and tumor progression. Cancer Res 2001;61:2129-2137.

31 Iizuka N, Oka M, Yamada-Okabe H, et al. Molecular signature in three types of hepatocellular carcinoma with different viral origin by oligonucleotide microarray. Int J Oncol 2004;24:565-574.

32 Clurman BE, Hayward WS. Multiple proto-oncogene activations in avian leukosis virus-induced lymphomas: evidence for stage-specific events. Mol Cell Biol 1989;9:2657-2664.

33 Eis PS, Tam W, Sun L, et al. Accumulation of miR-155 and BIC RNA in human B cell lymphomas. Proc Natl Acad Sci USA 2005;102:3627-3632.

34 Tam W, Hughes SH, Hayward WS, et al. Avian bic, a gene isolated from a common retroviral site in avian leukosis virus-induced lymphomas that encodes a noncoding RNA, cooperates with c-myc in lymphomagenesis and erythroleukemogenesis. J Virol 2002;76:4275-4286.

35 Nakopoulou L, Lazarisa AC, Kavantzasa N, et al. DNA topoisomerase II-alpha immunoreactivity as a marker of tumor aggressiveness in invasive breast cancer. Pathobiology 2000;68:137-143.

36 Theocharis SE, Margeli AP, Klijanienko JT, et al. Metallothionein expression in human neoplasia. Histopathology 2004;45:103-118.

37 Deng DX, Chakrabarti S, Waalkes MP, et al. Metallothionein and apoptosis in primary human hepatocellular carcinoma and metastatic adenocarcinoma. Histopathology 1998;32:340-347. 
38 Endo T, Yoshikawa M, Ebara M, et al. Immunohistochemical metallothionein expression in hepatocellular carcinoma: relation to tumor progression and chemoresistance to platinum agents. J Gastroenterol 2004;39:1196-1201.

39 Ebara M, Fukuda H, Hatano R, et al. Relationship between copper, zinc and metallothionein in hepatocellular carcinoma and its surrounding liver parenchyma. J Hepatol 2000;33:415-422.

40 Quaife CJ, Cherne RL, Newcomb TG, et al. Metallothionein overexpression suppresses hepatic hyperplasia induced by hepatitis B surface antigen. Toxicol Appl Pharmacol 1999;155:107-116.

41 Suzuki JS, Nishimura N, Zhang B, et al. Metallothionein deficiency enhances skin carcinogenesis induced by 7,12-dimethylbenz[a]anthracene and 12-O-tetrade- canoylphorbol-13-acetate in metallothionein-null mice. Carcinogenesis 2003;24:1123-1132.

42 Waalkes MP, Liu J, Kasprzak KS, et al. Hypersusceptibility to cisplatin carcinogenicity in metallothioneinI/II double knockout mice: production of hepatocellular carcinoma at clinically relevant doses. Int J Cancer 2006;119:28-32.

43 Datta J, Majumder S, Kutay $\mathrm{H}$, et al. Metallothionein expression is suppressed in primary human hepatocellular carcinomas and is mediated through inactivation of CCAAT/enhancer binding protein alpha by phosphatidylinositol 3-kinase signaling cascade. Cancer Res 2007;67:2736-2746.

44 Pang RW, Poon RT. From molecular biology to targeted therapies for hepatocellular carcinoma: the future is now. Oncology 2007;72(Suppl 1):30-44.

Supplementary Information accompanies the paper on Modern Pathology website (http://www.nature.com/ modpathol) 